\title{
Journal of Perioperative and Critical Intensive Care Nursing
}

\section{Anthropometric Measurement of Patients Admitted to an Intensive Care Unit}

Wai Keung Cheung ${ }^{*}$, Ying Chu Choi, lai Sheung Chau and lon leng Laurinda Mak

Department of Nursing Practice, School of Nursing, University of Hong Kong, Hong Kong

${ }^{*}$ Corresponding author: Wai Keung Cheung, RN, MN, MSc App. Epi., MSc Public Health, Assistant Professor, Department of Nursing Practice, School of Nursing, The University of Hong Kong, Hong Kong, Tel: +852 98698701; Fax: +852 2872 6079; E-mail: alessi@hku.hk

Received date: Mar 14, 2016; Accepted date: Apr 05, 2016; Published date: Apr 12, 2016

Copyright: (C) 2016 Cheung WK, et al. This is an open-access article distributed under the terms of the Creative Commons Attribution License, which permits unrestricted use, distribution, and reproduction in any medium, provided the original author and source are credited.

\begin{abstract}
Background: Visual estimation is the usual method that many healthcare professional use to estimate the body weight of patients in the Intensive Care Unit (ICU), but this method has been shown to be significantly inaccurate. This study aimed to explores the feasibility of using fibula length to estimate the acute body weight of patients admitted to an ICU.

Methods: The study collected all ICU records in which patient's body weight was estimated by the equation:

Males: $153.1-(0.26 \times$ age $)-11+(1.05 \times$ fibular length $)$

Females: $153.1-(0.26 \times$ age $)-22+(1.05 \times$ fibular length $)$.

The body weight(BW) then estimated by: $B W=20 \times$ [estimated body height (in metres)]2. The degree of agreement between the estimated body weight by anthropometric measurement methods and the actual recorded body weight in the patient's medical record within the four weeks immediately before ICU admission were assessed by the Bland-Altman plot.
\end{abstract}

Results: Paired sample t-tests showed there were statistically significant differences between the patient's estimated and actual height and weight ( $p$-value $=0.0001$ for both).

Conclusions: The study found the use of fibula length alone had a similar percentage of bias when compared with visual estimation by healthcare professionals to estimate the actual body weight of the patients admitted to ICU.

Keywords: Critical care nursing; Clinical research; Evidence-based practice; Intensive care nursing; Nutrition

\section{Relevance to Clinical Practice} less

(1) The actual body weight of Asian patients was found to be $10 \%$

than the standard $70 \mathrm{~kg}$ male and $60 \mathrm{~kg}$ female estimates that most

healthcare professionals used in clinical settings.

(2) The actual body weight of Asian patients was less than that of Western

populations by $30 \%$ for men and $25 \%$ for women, on average.

(3) The use of standard $70 \mathrm{~kg}$ male and $60 \mathrm{~kg}$ female estimates cannot be

applied universally in all ICU settings, as different ethnicity of the patient

may have different BMI cutoff.

(4) The use of fibula length alone had a similar percentage of bias when

compared with visual estimation by healthcare professionals.

\section{Introduction}

Assessing the body weight and height of critically ill patients in the intensive care unit (ICU) is often essential, but a real challenge for the healthcare professionals concerned $[1,2]$. This is especially true for physicians, nurses and dieticians, as many forms of clinical management - mechanical ventilation by tidal volume per kilogram per breath, antibiotics, nutritional support, inotropes and the dosage of other drugs - all require the patient's body weight to inform management procedures. To estimate the patients' body weight, the usual ICU practice is simply visual estimation, but such methods have been shown to be significantly inaccurate with an error ranged from $11.4 \%$ to greater than $20 \%$ against measured values [3-5]. Different institutes or studies developed differnet equations, such as National Institutes of Health (NIH) ARDS Network (ARDSNet), actuarial table (ACTUARIAL) and Stewart (STEWAERT) predicted body weight equation, to predict body weight but significant potential differences existed between these equations [6]. The best bedside method to estimate patients' own body weight is probably simply to ask the patient [7-9], but this is not always feasible in an ICU as many patients are intubated, comatose, cannot recall their own body weight, have undergone organ removal during surgery, or their body-fluid components have been changed because of fluid resuscitation or disease progress, such as generalised oedema. 
To achieve safe and adequate nutrient support for critically ill patients, the body weight estimation error should be minimised. The study aimed to explores the feasibility of using fibula length to estimate the acute body weight of patients admitted to an ICU, approval for which has been obtained from the ethics committees of both the hospital and the University.

\section{Methods}

A retrospective review of ICU records was carried out in 2015, at a 26-bed unit spread over two wards on the same floor, a general ICU catering for both medical and surgical cases, including trauma, orthopaedic and neurosurgical patients. The turnover of patients was $1,800-2,000$, making it one of the largest ICUs in Hong Kong.

The data collection period ran from April 2012 to Aug 2013, with patients identified from the unit's admission book. Admissions of all kinds within this period totalled 2,400. Of the corresponding 2,400 sets of records in the admission book, 986 fulfilled the inclusion criteria but only 490 recorded that the fibular length had been measured and charted by the ICU nurse immediately after admission, when the height was estimated by the following formula [10]:

Males: $153.1-(0.26 \mathrm{x}$ age $)-11+(1.05 \mathrm{x}$ fibular length $)$

Females: $153.1-(0.26 \mathrm{x}$ age $)-22+(1.05 \mathrm{x}$ fibular length $)$

The inclusion and exclusion criteria were as follows:

\section{Inclusion criteria}

Adult ICU patients with nasogastric tube (NG) or oro-gastric (OG) feeding, with nutritional product equal to $1 \mathrm{Kcal} / \mathrm{ml}$.

\section{Exclusion criteria}

- Paediatric patients as there are changes in weight and height with age as well as their relation to body fatness for children. BMI levels among children and teens need to be expressed relative to other children of the same sex and age [11]

- Pregnant patients as maternal Boady Mass Index (BMI) increase with parity [12]

- Patients currently under total supplemental parental nutrition

- Morbidly obese patients with estimated BMI > 30.0 which exceed the median BMI proposed in this study

- Malnutrition/prolonged fasting before ICU admission to avoid severe re-feeding syndrome if one of these four criteria is met:

- Estimated BMI $<16.0$ which below the proposed median BMI in this study

- Unintentional significant body weight loss $>15 \%$ within last three to six months (reliable history from patient or informant or parent ward)

- Clinically cachexic-looking, or alcoholic with features of significant muscle wasting

- Patients under jejunostomy or PEG feeding which didn't fulfill the inclusion criteria

- Patients with acute pancreatitis which require fasting

- Patients with gastrointestinal problems, including post-operative gastrointestinal surgery which cannot initiate the enteral nutrition immediate postoperatively.

Since comorbidity, mortality and body composition data consistently support the use of lower Body Mass Index (BMI) cutoffs in
Chinese than those in whites [13], the body weight was estimated indirectly by using the median value of the BMI (range=18.5-23, midvalue $=20.0$ ) according to the Malnutrition Universal Screening Tool for Asian [14]:

Estimated body weight $=20 \mathrm{x}$ [estimated body height (in metres) $] 2$

The estimate is then compared with the patient's actual body weight, retrieved from medical records within a month before admission to the ICU.

Finally, the estimate target feeding rate was calculated by using the conversion table, and started at $30 \mathrm{ml} / \mathrm{hr}$ until the target rate was reached.

The remaining cases either fulfilled the exclusion criteria or did not fulfil the inclusion criteria and as a result, according to the protocol, enteral feeding did not start and dieticians were consulted.

The primary outcome was the degree of agreement between the estimated body weight by anthropometric measurement methods and the actual recorded body weight in the patient's medical record within the four weeks immediately before ICU admission.

Data analysis included descriptive statistics of different variables (mean, median, IQR and SD), depicting the distribution of these variables. A Bland-Altman plot was employed to assess whether the anthropometric method of estimating body weight was comparable to traditional weighing methods, and a paired sample t-test was employed to check for any significant difference between these two methods. All calculations were carried out by means of SPSS V23.

\section{Results}

A total of 2,400 sets of records in the admission book found within the data collection period, 986 fulfilled the inclusion criteria but only 490 recorded that the fibular length had been measured and charted by the ICU nurse immediately after admission and hence included in this study.

The median age of patients was 60.0 (range 18-97) and the male-tofemale ratio was 290:200 (approximately 1.45:1). The mean actual body weight was $53.58 \mathrm{~kg}(\mathrm{SD}=6.22)$. By dividing male from female patients, the mean actual body weight and height of (A) male and (B) female patients retrieved from medical records within the month before admission were found to be (A) $63.4 \mathrm{~kg}(\mathrm{SD}=11.59)$ and 1.65 metres $(\mathrm{SD}=0.076)$, and $(\mathrm{B}) 54.89 \mathrm{~kg}(\mathrm{SD}=11.20)$ and 1.53 metres $(S D=0.061)$. However, only 290 patients had their actual body weight measured within the month before admission, and only 227 patients had their height measured during that period.

The mean duration of stay was 4.54 days (range 0.05-85.76). 455 patients were only admitted once (92.9\%), 31 (6.3\%) were admitted twice and four $(0.8 \%)$ three times during the study period. 62 patients $(12.7 \%)$ died there.

332 patients $(68.0 \%)$ had had an operation immediately before admission. 221 of these operations were of the emergency type (66.57\%), with the remainder elective $(n=109,33.43 \%)$.

\section{Estimated body height and weight by anthropometric methods}

The measured mean fibula length was $39.09 \mathrm{~cm}(\mathrm{SD}=4.81)$, the mean height estimated by measuring fibula length was 1.63 metres $(\mathrm{SD}=0.095)$, and the estimated body weight using $\mathrm{BMI}=20.0$ was 53.58 
$\mathrm{kg}(\mathrm{SD}=6.22)$. Paired sample t-tests showed there were statistically significant differences between (1) the patient's estimated and actual height ( $\mathrm{p}$-value $=0.0001,95 \%$ C.I. $0.017,0.036$ ) and (2) the estimated and actual body weight ( $\mathrm{p}$-value $=0.0001,95 \%$ C.I. $-7.88,-5.36$ ). The mean difference between (3) estimated and actual height was 0.2649 metres $(\mathrm{SD}=0.7591)$ and (4) estimated and actual weight was $-6.62 \mathrm{~kg}$ $(\mathrm{SD}=10.88)$.

\section{Degree of agreement in body weight measurement between anthropometric method and weighing scales}

To assess the degree of agreement, many studies take the productmoment correlation coefficient $(r)$ between the results of two measurement methods as an indicator of agreement [15]. However, the correlation process investigates the relationship between one variable and another and not the differences, and so it is not recommended as a method of assessing the comparability of different methods. For this reason, the degree of agreement was assessed by a Bland-Altman plot $[15,16]$. To fulfil the requirements for employing a Bland-Altman plot [15], the normal distribution of the differences between the two methods was verified by the Kolmogorov-Smirnov test (pvalue $=0.096$ ). Although measuring body weight by scales is a standard or reference method, the true value of the patient's body weight on admission to the ICU was not known and the mean of the two measurements was the best estimate we had. In addition, using scales is controversial, as a plot of difference against a 'standard measurement' will always appear to show a relation between the difference and magnitude when there is none [16]. We therefore chose the mean of the two measurements for the $\mathrm{x}$-axis. Figure 1 shows the plot of differences between method $\mathrm{A}$ (anthropometric, using $\mathrm{BMI}=20.0$ ) and method B (scales) vs. the mean of the two measurements.

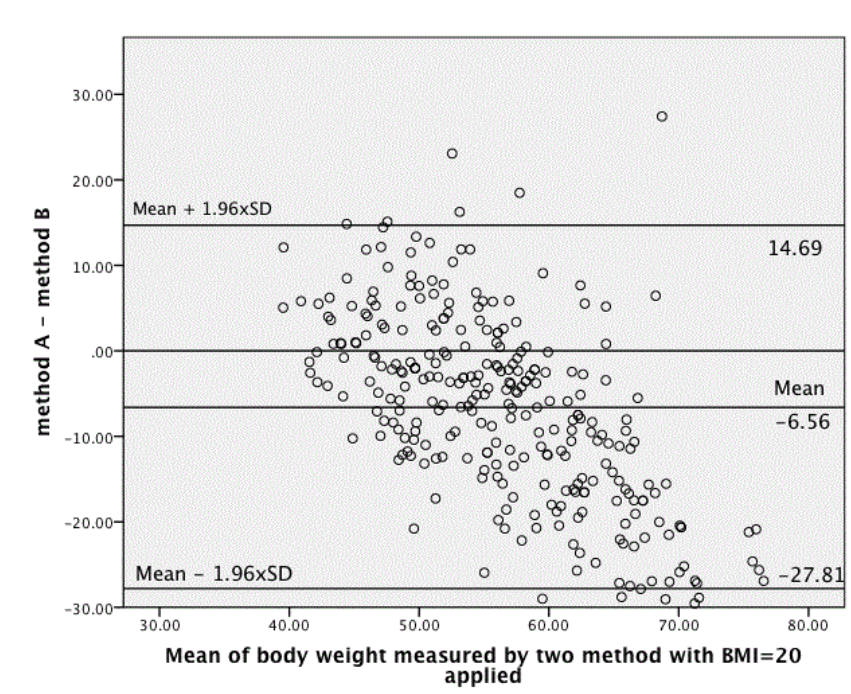

Figure 1: Plot of differences between method A (anthropometric, using $B M I=20.0$ ) and method B (scales) vs. the mean of the two measurements.

From Figure 1, it shows that the bias was $-6.56 \mathrm{~kg}$ and the range of agreement was $14.69 \mathrm{~kg}$ to $-27.81 \mathrm{~kg}$ (mean +/- $1.96 \mathrm{x} \mathrm{SD)}$ ). The best way to use the B\&A plot would be to define a priori limits of maximum acceptable differences (the limits of agreement expected), based on biologically and analytically relevant criteria, and then to produce statistics to see if these limits are exceeded or not. Before the implementation of the project, we did not define any limits of expected agreement, as we wanted to minimise the estimation so that it was as close to actual body weight as possible.

\section{Discussion}

This study describes the possibility of using fibula length to estimate indirectly the acute body weight of patients admitted to an ICU, with the aim of achieving safe and adequate nutrient support for this group of patients by better estimating their weight.

The actual body weight of male and female patients was $63.40 \mathrm{~kg}$ and $54.89 \mathrm{~kg}$ respectively, less than the standard $70 \mathrm{~kg}$ male and $60 \mathrm{~kg}$ female estimates that most healthcare professionals were using in clinical settings when measuring acute body weight was not feasible. In another study, the actual body weight of patients was estimated to be much higher $-81.8 \mathrm{Kg}$ for men and $68.8 \mathrm{~kg}$ for women [5]. The difference may due to the fact that average men and women in Western populations are much taller and more heavily built, while the subjects in the present study were Asians, who are normally smaller in build and weight. This is reflected in the greater average height of men and women in the other study when compared with ours (men: 1.72 vs. 1.65metres, and women: 1.59 vs. 1.53 metres) [5], which support the findings in other study that the use of lower BMI cutoffs in Chinese than those in whites [13].

Paired sample t-tests of the estimated and actual height of the patient, and the estimated and actual body weight revealed significant differences, with a mean height difference of 0.2649 metres and mean weight difference of $6.62 \mathrm{~kg}$. What this means is that the anthropometric method used in this study overestimated the average patient's height by 0.26 metres $(\mathrm{SD}=0.7591)$ and underestimated the weight by $6.62 \mathrm{~kg}(\mathrm{SD}=10.88)$, which means nutrition support based on the estimated body weight by using this method cannot provide adequate nutrition to the patient and may associated with poor clinical outcomes [17]. However, we cannot conclude that the method was inaccurate in estimating the actual body weight of the patients admitted to the ICU, because the reference weight we selected was the actual body weight measured by scales within the month before admission. Besides, body weight may of course change when the patient has a severe illness or has had any major surgery where internal organs may have been removed. However, the method employed in this study did not consider the effects of surgery or disease severity, such as an APACHE score, and this may have caused inaccurate estimates.

On the other hand, since skeleton height remains relatively constant after puberty, we would not expect there to be any great changes in a patient's height within one month, irrespective of any surgery or severity of illness. The mean difference between the height produced by the anthropometric method and acute patient height was 0.26 metres $(\mathrm{SD}=0.7591)$, which means that using fibular length may overestimate the height by 1.75 metres or underestimate it by -1.23 metres, as maxima (means $+/-1.96 \mathrm{x} \mathrm{SD}$ ). Since the standard variation was so large and the paired sample t-test had already shown there was a significant difference between the estimated and actual height of the patient, we may conclude that the use of fibula length alone to estimate patients' height when admitted to the ICU is not feasible. In fact, one similar study, published in 2011, used tibial length to estimate the height and weight of the patient and also showed great variation, with estimates deviating by $33.9 \%$ and $36.3 \%(>+/-5 \mathrm{~kg}$ ) from the ideal 
body weight of the males and females attending the emergency department [9].

To assess the degree of agreement, a Bland-Altman (B\&A) plot was employed. The B\&A plot does not say if the agreement is sufficient or suitable for use by one method or another. It simply quantifies the bias and a range of agreement within which $95 \%$ of the differences between one measurement and another are included [15]. With results showing a $12.24 \%$ bias, similar to other studies using visual estimation by a physician (10.58\%) or nurse (10.80\%) [5] or tibial length [9], it seems that visual estimation already serves the same purpose as the method that we employed in this project, with a similar estimation error. In addition, the range of agreement was very large which against the use of fibula length alone to estimate the acute body weight of patients admitted to the ICU.

\section{Limitations}

The only available acute body weight reference was that of patients measured within the month before admission, which might have undergone drastic change within that month, especially if the patient had just had or suffered from a severe disease, such as severe sepsis causing haemodynamic instability with a large amount of fluid required to stabilise blood pressure. Another limitation was that the fat, muscle and fluid components of a body may be different for people of the same height and age, and hence their weight may differ. For example, the fat and muscle components of a 30-year-old man who does regular bodybuilding exercise may be very different from those of a person of the same height and age who does not. The method used in this study without considering these factors might further under- or over-estimate the actual body weight of patients admitted to the ICU.

\section{Conclusion}

The study explores the feasibility of using fibula length to estimate the acute body weight of patients admitted to an ICU, and added certain facts to the present body of knowledge about acute body weight estimation in the ICU: (1) the actual body weight of Asian patients was found to be $10 \%$ less than the standard $70 \mathrm{~kg}$ male and $60 \mathrm{~kg}$ female estimates that most healthcare professionals used in clinical settings; (2) the actual body weight of Asian patients was less than that of Western populations by $30 \%$ for men and $25 \%$ for women, on average; (3) the use of standard $70 \mathrm{~kg}$ male and $60 \mathrm{~kg}$ female estimates cannot be applied universally in all ICU settings, as different ethnicity of the patient may have different BMI cutoff; (4) the use of fibula length alone had a similar percentage of bias when compared with visual estimation by healthcare professionals. Despite many studies applying different methods to estimate patients' acute body weight, patient self-reporting still appears to be the most accurate. However, this method does not necessarily work in an ICU, where most patients are intubated, sedated, comatose or simply cannot stand or sit on traditional scales to have their actual weight measured. We suggest further exploration of any other methods that might minimise the body weight estimation error. In the meantime, we further suggest upgrading all ICU beds by adding a weighing function of some kind, in order to achieve safe and adequate nutrient support for critically ill patients.

\section{What is known about this topic}

- To estimate the patients' body weight, the usual ICU practice is simply visual estimation, but such methods have been shown to be significantly inaccurate. The error can be as much as or greater than $20 \%$ against measured values.

- Patients' own estimates of their body weight are more accurate than those of healthcare staff.

\section{What this paper adds}

- The actual body weight of Asian patients was found to be $10 \%$ less than the standard $70 \mathrm{~kg}$ male and $60 \mathrm{~kg}$ female estimates that most healthcare professionals used in clinical settings;

- The actual body weight of Asian patients was less than that of Western populations by $30 \%$ for men and $25 \%$ for women, on average;

- The use of standard $70 \mathrm{~kg}$ male and $60 \mathrm{~kg}$ female estimates cannot be applied universally in all ICU settings, as different ethnicity of the patient may have different BMI cutoff;

- The use of fibula length alone had a similar percentage of bias when compared with visual estimation by healthcare professionals.

\section{References}

1. Diacon AH, Koegelenberg CF, Klusmann KJ, Bolliger CT (2006) Challenges in the estimation of tidal volume settings in critical care units. Intensive Care Med 32: 1670-1671.

2. Freitag E, Edgecombe G, Baldwin I, Cottier B, Heland M (2010) Determination of body weight and height measurement for critically ill patients admitted to the intensive care unit: A quality improvement project. Aust Crit Care 23: 197-207.

3. Bloomfield R, Steel E, MacLennan G, Noble DW (2006) Accuracy of weight and height estimation in an intensive care unit: Implications for clinical practice and research. Crit Care Med 34: 2153-2157.

4. Leary TS, Milner QJ, Niblett DJ (2000) The accuracy of the estimation of body weight and height in the intensive care unit. Eur J Anaesthesiol 17: 698-703.

5. Maskin LP, Attie S, Setten M, Rodriguez PO, Bonelli I, et al. (2010) Accuracy of weight and height estimation in an intensive care unit. Anaesth Intensive Care 38: 930-934.

6. Linares-Perdomo O, East TD, Brower R, Morris AH (2015) Standardizing Predicted Body Weight Equations for Mechanical Ventilation Tidal Volume Settings. Chest 148: 73-78.

7. Lin BW, Yoshida D Fau - Quinn J, Quinn J Fau - Strehlow M, Strehlow M (2009) A better way to estimate adult patients' weights. Am J Emerg Med 29: 1060-1064.

8. Spicer K, Gibson P, Bloe C, Cross SJ, Leslie SJ (2009) Weight assessment in cardiac patients: implications for prescription of low molecular weight heparin. Postgrad Med J 85: 124-127.

9. Stehman CR, Buckley RG, Dos Santos FL, Riffenburgh RH, Swenson A, et al. (2011) Bedside estimation of patient height for calculating ideal body weight in the emergency department. J Emerg Med 41: 97-101.

10. Auyeung TW, Lee JS (2001) Estimation of height in older Chinese adults by measuring limb length. J Am Geriatr Soc 49: 684-685.

11. CDC (2015) About child and teen BMI.

12. Johansson K, Hutcheon JA, Stephansson O, Cnattingius S, Ugwuja EI, et al. (2016) Pregnancy weight gain by gestational age and BMI in Sweden: a population-based cohort study. Am J Clin Nutr.

13. Cho JH, Choi JH, Suh JD, Ryu S, Cho SH, et al. (2015) Comparison of Anthropometric Data Between Asian and Caucasian Patients With Obstructive Sleep Apnea: A Meta-Analysis Lower BMI cutoffs to define overweight and obesity in China.

14. WHO (2004) Appropriate body-mass index for Asian populations and its implications for policy and intervention strategies. Lancet 363: 157-163.

15. Giavarina D (2015) Understanding Bland Altman analysis. Biochem Med (Zagreb) 25: 141-151. 
Citation: Cheung WK, Choi YC, Chau IS, Mak IIL (2016) Anthropometric Measurement of Patients Admitted to an Intensive Care Unit. J Perioper Crit Intensive Care Nurs 2: 113. doi:10.4172/jpcic.1000113

Page 5 of 5

16. Bland JM, Altman DG (1986) Statistical methods for assessing agreement between two methods of clinical measurement. Lancet 1: 307-310.
17. Lew CC, Yandell R, Fraser RJ, Chua AP, Chong MF, et al. (2016) Association Between Malnutrition and Clinical Outcomes in the Intensive Care Unit: A Systematic Review. 